\title{
تطبيق الإستراتيجية المعرفية مع تقنية ” أخذ الملاحظات “ لتزقية قدرة الطلبة فى فهم النصوص العربية بجامعة معارف فضة العلماء الإسلامية مترو لمبونج
}

\author{
محمد سيف الله \\ muhammadsyaifullah121285@gmail.com \\ جامعة معارف الإسلامية فضة العلماء مترو لمبونج \\ ملخص : يعتمد هذا البحث على قلة القدرة على القراءة في اللغة العربية لدي الطلبة. يحدث هذا بسبب أنشطة التعلم التي تميل إلى مملة واستعمال \\ الإستراتيجية سواء في كل اجتماع، ولذلك يجعل الطلبة نسيانا عن المواد التي قد تم تعليمها. ويهدف هذا البحث بهدف وصف تنفيذ التعلم بقراءة \\ النصوص العربية بالاستراتيجية المعرفية مع تقنية أخذ الملاحظات ووصف نتيجة زيادة قدرة لطلبة في فهم النصوص العربية بجامعة معارف الإسلامية \\ هضة العلماء مترو لمبونج من خلال استخدام الإستراتيجية المعرفية مع تقنية أخذ الملاحظات. ونوع هذا البحث ببحث الإجرائي، هي التخطيط \\ والتنفيذ والملاحظة والتفكير. يتم إجراء هذا البحث بدورتين، في كل دورة اجتماعان. الأدوات المستخدمة هي الأسئلة الاختبار وأوراق للمراقبة وأدلة \\ للمقابلة. ظهرت النتائج: (1) زيادة قدرة لطلبة فن فهم النصوص العربية مع تقنية أخذ الملاحظات. في الإجتماع الأولى من الدورة الأولى التي عرفتها \\ في جلسات القراءة الجهرية، كان معظم الطلبة أقل مجموعًا في التصويت، ولكن في الاجتماع الثاني زاد. (Y) يشعر الطلبة أيضًا بالمساعدة في فهم \\ محتويات النصوص العربية مع تقنية أخذ الملاحظات لأن هذه التقنية بسيطة جدًا وسهلة التطبيق(ب)من البيانات التي تم الحصول عليها، من \\ المعروف أن قدرة لطلبة فى فهم النصوص العربية قد ازدادت، أي قبل إجراء نتائج تعلم الطلبة في المتوسط فقط هـ\% بعد فاية الإجراء في الدورة \\ الأولى والمعدل قدره 9V\%، في الدورة الأولى والمعدل قدره 7. \%.\%. \\ الكلمات المفتاح : الإستراتيجية المعرفية، تقنية أخذ الملاحظات، فهم النصوص العربية
}

\begin{abstract}
Abstrak: Penelitian ini berlatar belakang pada kurangnya kemampuan membaca teks bahasa Arab mahasiswa. Hal ini disebabkan oleh kegiatan pembelajaran yang cenderung membosankan dan strategi yang selalu sama pada setiap pertemuan sehingga membuat mahasiswa mudah lupa dengan materi yang telah disampaikan. Penelitian ini dilaksanakan dengan tujuan untuk Mendeskripsikan pelaksanaan pembelajaran membaca teks bahasa Arab dengan strategi kognitif teknik taking notes dan mendeskripsikan hasil peningkatan penguasaan membaca teks bahasa Arab mahasiswa Pendidikan Bahasa Arab IAIM NU Metro Lampung melalui penggunaan strategi kognitif dengan teknik taking notes. Rancangan penelitian menggunakan penelitan tindakan kelas (Classroom Action Research), yaitu perencanaan, pelaksanaan, observasi dan refleksi. Penelitian ini dilakukan dengan 2 siklus, setiap siklusnya terdapat 2 pertemuan. Instrumen yang digunakan adalah soal tes, lembar observasi dan pedoman wawancara. Hasil penelitian menunjukan bahwa: (1) kemampuan mahasiswa dalam memahami teks bahasa Arab dengan teknik taking notes mengalami peningkatan. Pada pertemuan pertama siklus I diketahui pada sesi membaca nyaring sebagian besar mahasiswa kurang total dalam mengeluarkan suara, namun di pertemuan ke dua sudah mengalami peningkatan. (2) mahasiswa juga merasa terbantu dalam memahami isi teks bahasa Arab dengan teknik taking notes karena teknik ini sangat simpel dan mudah diterapkan. (3) Dari data yang di dapatkan diketahui bahwa kemampuan mahasiswa dalam memahami teks bahasa Arab mengalami peningkatan, yakni
\end{abstract}


sebelum dilakukan tindakan hasil belajar mahasiswa rata-rata hanya $45 \%$ setelah akhir tindakan pada siklus I rata-rata $79 \%$, dan siklus II rata-rata $86 \%$.

Kata Kunci : memahami teks bahasa Arab, strategi kognitif, teknik taking notes

شاملة لتقديم اللغة بشكل منتظم على أساس

\section{مقدّمة}

التقريب المحدد. في حين أن التقنية هي

التعليم الجيد يمكن أن يدعم التنمية في

أنشطة محددة يتم تنفيذها في الفصول

جميع المجالات. لذا، يحتاج التعليم إلى

الدراسية، في انسجام مع الطرق والمقاربات

الحصول على اهتمام كبير حتى نتمكن من

المختارة. وهكذا، يكون التقريب بديهيًا،

اللحاق بركب العلوم والتكنولوجيا التي نحتاج

والطريقة إجرائية، والتقنية هي عملية

إليها على وجه السرعة لتسريع التنمية.

شفافية' . م ك.

تعتبر التقنية أخذ الملاحظات أحد التقنية

المناسبة لإتقان تعلم اليوم وخاصة في منحة

لذلك، يجب أن يجصل التعليم الجيد على

القراءة لفهم محتويات النصوص العربية.

اهتمام جاد من الحكومة. أصبح عالم التعليم

الآن هو المحور الرئيسي للحكومة والعالم

الدولي، خاصة في غياب تباين استراتيجيات

وذكرت نور هداية وآخرون، أن تقنية أخذ

التعلم في عملية التعليم والتعلم.

الملاحظات هي عملية مهمة جدًا في أنشطة

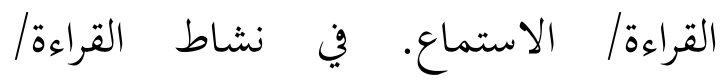

الاستماع لقصة على سبيل المثال يمكن

في تعليم اللغة، هناك ثلاثة مصطلحات

تحتاج إلى فهمها بشكل مناسب ومفهوم،

وهذا هو التقريب والطريقة والتقنية. التقريب

1 Ahmad Fuad Effendy, Metodologi

Pengajaran Bahasa Arab, (Malang: Misykat, 2009), hal. 8

2 Nurhidayati, \& Anisah, Strategi Pembelajaran Bahasa Arab Untuk Anak,

هو مجموعة من الافتراضات المتعلقة بطبيعة

اللغة، وتعليم اللغة والتعلم. الطريقة هي خطة (Malang: Fakultas Sastra UM, 2005), hal. 35 
الإستراتيجية المعرفية لجاني في يامين ،

هي قدرة الفرد على التفكير، وحل المشكلات، و واتخاذ القرارات. تؤدي قدرة

الإستراتيجية المعرفية على عمليات تفكير

فريدة في تحليل المشكلات وحلها وفي اتخاذ

القرارات. القدترة والتفرد في التفكير كسيطرة

تنفيذية، أو تدعوا بالتحكم عالي المستوى،

أي تحليل حاذق ومُلائم ودقيق. بمكن

ملاحظة ذلك في حياة السياسة الإندونيسية اليوم، أوليك الذين لديهم قدرة معرفية عالية يمكنهم حل المشكلة بسهولة، ولكن من السهل عكس الحقائق والمفاهيم ومبادئ المصالح السياسية التي تدعموفها، والعكس بالعكس قدرقم الإدراكية المنخفضة خذ

$$
\text { انفراجة فقط كحزمة للمشاركة. }
$$

الإستراتيجية المعرفية هي القدرة التي تنظم

كيفية إدارة الطلبة للتعلم، عند التذكر

والتفكير، وهي أيضًا عملية التحكم أو إدارة
للمعلمين إعداد جدول يحتوي على أشياء مهمة من القصة التى قد استمعت أو قرأت عليها. الإطار النظري الاستراتيجية المعرفية

يصف أكسفورد في نور هداية ، نظرة عامة على الاستراتيجيات تعليم اللغة المصنفة في استراتيجيتين: الاستراتيجية المباشرة والاستراتيجية غير المباشرة (direct strategies) (indirect strategies). تشمل الاستراتيجية المباشرة إلى ثلاث استراتيجيات للتعلم: الإستراتيجية الذاكرة (strategies memory)؛ والاستراتيجية المعرفية (strategies cognitive)؛ compensation ) والإستراتيجية التعويضية Strategies ) واحدة من استراتيجيات التدريس المرتبطة بالاستراتيجية المباشرة هي الاستراتيجية المعرفية.
4 Martinis Yamin, Paradigma Pendidikan Konstruktivistik, (Jakarta: Gaung Persada Press, 2008), hal. 5

\footnotetext{
Pembelajaran......... hal. 29
} 
المهارات الفكرية، ولا تتأثر الإستراتيجية بشكل كبير بتنفيذ التعليم، دقيقة بدقيقة. يمكن تطوير مهارات الاستراتيجية المعرفية حتى درجة معينة بشكل أفضل مع التعليم الرسمي، ويتعلم الطلبة ويتطورون بأنفسهم، ويفكرون في اكتساب الاكتفاء الذاتي .

\section{تقنيات التعلم مع الاستراتيجية المعرفية}

أوضحت نور هداية وآخرون ، أن "هذه

الاستراتيجية تمكن اتباعها من خلال تقني

(1) التدريب (racticing) (r) الإستقبال

receiving and sending ) والإرسال الرسائل

(r) ؛ (messeges

(analyzing and reasoning)

المفاهيم أو هياكل للمواد المدخلة أو بنية

creating structure for input ) إنشاء مخرجات

." (and output

في هذا البحث فقط المكرسة لخلق مفهوم
العناصر الفاعلة. تؤثر الاستراتيجية المعرفية على انتباه الطلبة إلى المنبهات، وأنظمة ترميز الطلبة، ومكدسات المعلومات المخزنة في الذاكرة. تؤثر هذه السعة أيضًا على الإستراتيجية الطلبة في العثور على الأشياء المخزنة وفي تنظيم الاستجابات و وإعادة اكتشافها. يقول غدني في يامين ، أن الاستراتيجية المعرفية تشبه سلوكيات الإدارة الذاتية.

الإستراتيجية المعرفية هية الاستدلالية أو الاستقرائية. بحربة مع الأشياء أو الأحداث، وهناك واحد يسعى للحصول على تفسير لظاهرة معينة من الحث. على النقيض من المعلومات الشفهية والمهارات الفكرية، والتي ترتبط مباشرة بالمحتوى. الهدف من الاستراتيجية المعرفية هو عملية التفكير

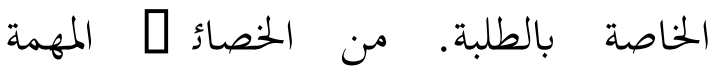
الأخرى للاستراتيجية المعرفية هي عكس

\footnotetext{
6 Martinis Yamin, Paradigma Pendidikan....... hal. 6 
إلى حد ما لاستخدامها على مستوى عال أو هيكل للمواد المدخل أو مواد الإخراج من المهارة (مثل الاستماع إلى تفسيرات (إنشاء الهيكلية للإدخال والإخراج). تساعدة هذه الاستراتيجية الطلبة على تنظيم المعلومات المعلم). هناك العديد من الطرائق المختلفة لأخذ أو المفاهيم من اللغة التي تتعلمونها وإظهار الملاحظات، أبسط أشكالها/سهلة الاستخدام

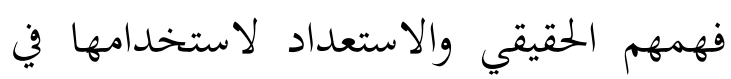
التحدث والكتابة. هناك ثلاثة أساليب لتنفيذ والأكثر شيوعا هي الملاحظات الخام التي لا تزال غير منظمة وغيرت. من أجل استخدام

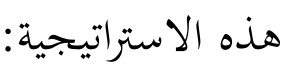
السجلات الأولية، يجب على الطلبة إعادة تنظيم سجلاته باستخدام أنظمة أخرى في أقرب وقت مككن. أفضل الطريقة الأخرى هي استخدام "قائمة التسوق" أو "تشكيل T" كما في المراحل المبكرة من القضاء على السجل الخام. وتتمثل ميزة الإستخدام أحد هذه الأدوات في مساعدة الطلبة على الإدارة ما يسمعونه أثناء الاستماع، مع تعزيز الفهم الأصلي والقدرة على الجمع بين المعلومات

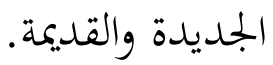
(1). تقنية أخذ الملاحظات(taking notes). (r) (summarizing) r) (ب ). وضع العلامات(hhlighting) . جعل تقنية أخذ الملاحظات أوضح أكسفورد ، أن أخذ الملاحظات في هذه المذكرة هي الإستراتيجية المهمة جدًا

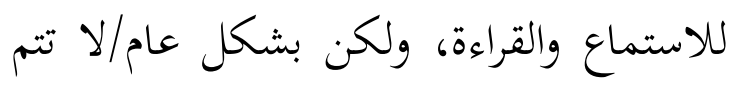
تعليم الطلبة بشكل عام لاستخدامها بشكل مثالي. تقديم الملاحظات هي وسيلة متقدمة يستخدم التنسيق T المادة نفسها كما في قائمة التسوق. يشبه هذا التنسيق قائمة

$8 \quad$ R. L.Oxford, Language Learning Strategies, What Every Teacher Should Know, (USA: Newbury House Publishers, 1990), hal. 8688 
التسوق، ولكن في هذه الحالة، يُسمح للطلبة المحمول ذو الأوراق السائبة هي أفضل باستخدامها/ توفير المسافة إلى الورقة المذكرة الطريقة. بالنسبة للطلبة الذي يكتبون شيئًا بطريقة أكثر فعالية. تتمثل الخطوة الأولى في كبيرًا في اللغة المستهدفة، من المفيد وضع رسم T كبيرًا على الورق عبر الورق، ثم كتابة الفكرة في أقرب وقت ممكن في الرأس. لذلك، الموضوع/العنوان الرئيسي أعلى الخط، على بيجب أن يتم الاحتفاظ دفنر في هيع الجانب الأيسر من الخط العمودي في كتابة الأوقات. الفئات/ الموضوعات الأساسية التي تمت وفقاً لنور هيداية وآخرون ، فإن أخذ مناقشتها بالفعل، على الجانب الآخر يتم الملاحظات عملية مهمة جداً في أنشطة كتابة الخطوط العمودية اليمنى في أقسام القراءة/الاستماع. في نشاط القراءة/ الاستماع تفاصيل، أمثلة محددة، إجابات للأسئلة، أو قصة على سبيل المثال يمكن للمعلم إعداد جدول يمتوي على أشياء مهمة من القصة تعليقات. الإستمع أو القراءة. على سبيل المثال: تقنية أخذ الملاحظات في تعليم مهارة اللغة الجدول. مثال على أخذ الملاحظات يمكن دمج تقنيات التسجيل مع الأنشطة

$$
\text { الموضوع }
$$$$
\text { والمواد اللغوية العادية مثل العناصر الطبيعية في }
$$
تعليم اللغة. تتمثل الاستراتيجية الفوقية المعرفية إلى جانب أخذ الملاحظات في المنظمة التي يوجد بها حراسة للمفكرة لتضمين معلومات لغة جديدة وتطويرها. يجب أن تبقى ملاحظة بدقة في بعض الأماط، قد يكون الكمبيوتر Pembelajaran......... hal. 35 
لاكتساب عن العلم والمعرفة. بدون القدرة على القراءة، يمكن أن يقال إن البشر لا يستطيعون العيش في هذا العصر، لأن الحياة البشرية تعتمد بشكل كبير على العلوم التي تمتلكها، وذلك لاكتساب المعرفة بأن أحدهم من خلال القراءة. يقول غدمان في آل عبد الله ، أن القراءة هي نشاط لاستخلاص المعنى من الطباعة أثناء القراءة باستخدام اللغة

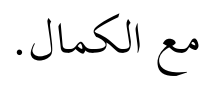
في وهذا العصر، يكون لدى الطلبة اهتماما أقل بالقراءة وخاصة قراءة الكتب المدرسية. لأن البعض منهم ليس لديهم الطريقة لقراءة، لذلك في وقت القراءة ينشأ شعور كسول، بالملل، و والنعاس. وفقا لنغراها ، هناك العديد من الأثثياء التي يمكن القيام بها من أجل الخلق جو لطيف للقراءة،

1 A. F. Al Abdullah, Asasu'Ta'lim Al Qira'ah Al Naqidah $\mathrm{Li}$ Al Thalabah $\mathrm{Al}$ Mutafawwiqu:n Aqliyyan, (Yordania: 'A:lim Al Kutub Al Hadits, 2007), hal. 8

$\begin{array}{llr}\text { Yang } & \begin{array}{c}\text { Nugraha, E. S. 2008. } \text { Cara }^{2} \text { Membaca } \\ \text { Menyenangkan }\end{array} \\ \text { (www.sekolahindonesia.com). } & \text { (online). }\end{array}$ الجدول هو دليل للطلبة لتسجيل أشياء

\begin{tabular}{l|l|l|l|l}
\hline مرة & مكان & & \\
& & & & \\
& & & & \\
\hline & & & & \\
\hline
\end{tabular}
مهمة في القصة. ليس المقصود من تقنية جعل هذه المذكرة لتدريب مهارة الكتابة ولكن يتم التأكيد في القدرة على فهم قراءة وفقاً لنور

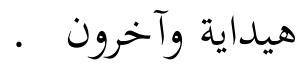

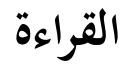
في القاموس الكبير المصور الإندونيسي، القراءة تأتي من الكلمة الأساسية للقراءة، القراءة تعني فهم ما هو مكتوب. القراءة هي واحدة من المهارات الأساسية الشائعة في تعليم اللغة. المهارات الأخرى هي مهارة الاستماع ومهارة الكام ومهارة الكتابة. القراءة هي واحدة من أهم العمليات $1 \quad$ Nurhidayati, \&
Pembelajaran......... hal. 36 
من بين أمور أخرى: اختيار الوقت الذي الحوافز، (r) القراءة المكثفة، (r) القراءة نعتقد أنه مناسب للقراءة، واختيار المكان الثابتة، (ع) القراءة بصوت عال، و (ه) (ه) المناسب والجو المناسب للقراءة، تأكد أن القراءة مع التوجيه.

هناك ثلاثة أنواع من القراءة في الحياة اليومية ينظر إليها من الغرض من عملية القراءة، من بين أمور أخرى. (1) القراءة على أهما تسلية بدون أرفاق للدماغ، (r) القراءة لاكتساب المعرفة، و (r) القراءة النقدية. وفقا لأفندي ، هناك عدة أنواع من أنشطة القراءة، من بين أمور أخرى؛ (1) القراءة الجهرية، (ץ) القراءة الصامتة، (ץ) القراءة السريعة، (ع) القراءة الاستمتاعية،

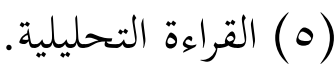

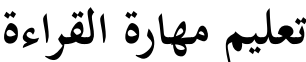

مهارة القراءة تحتوي على جانبين/ فهمين. أولا، تغيير رمز الكتابة إلى الصوت. Pengajaran......, hal. 158-164
موضع القراءة هو المكان المناسب، الأشياء التي تساعدنا عادة في القراءة.

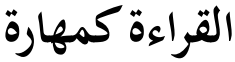

مهارة القراءة هي واحدة من المهارات الأساسية الأربع في التعليم اللغة، بالإضافة إلى مهارة الاستماع ومهارة الكلام ومهارة الكتابة. تنقسم القراءة كمهارة إلى ثلاثة أنواع

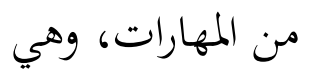
1). (1) مهارة التعرف على الكلمات

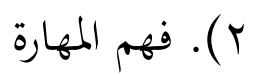

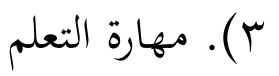

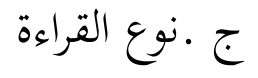
فيما يتعلق بنوع القراءة، يقسم الخولي القراءة إلى خمسة الأجزاء وهي: (1) القراءة Al Arabiyah, (Kairo: Jami:' Al Huquq, 1986), hal. 113 
الكلمات و والجملة والفقرة. هذه العناصر الثلاثة تدعم معا معنى مادة القراءة.

بشكل عام، الغرض من تعليم القراءة هو أن يقوم الطلبة بقراءة وفهم النصوص العربية. هناك العديد من الطرائق التي تمكن تطبيقها في تعليم القراءة، من بين الأمور الأخرى؛ (أ) الطريقة الحروف، (ب) الطريقة الصوتية، (ج) الطريقة المقطع، (د) الطريقة الكلمة، (هـ) الطريقة الجملة، و (و) الطريقة مختلطة .

نوع البحث الذي تم إجراؤه عبارة عن بحث الإجرائي (Research Classroom Action). ووفقًا لفيريا مادجا في رستينة ، فإن بحث الإجرائي هو كيف يمكن لمجموعة من المعلمين

1 Al Khuli, Asa:li:b Tadri:s 64l Lughah Al Arabiyah. hal. $107-112$

1 Nina Ristianah, Penerapdn Metode Pembelajaran Model Teams Games Tournament (TGT) Untuk Meningkatkan Hasil Belajar Akuntansi Siswa Kelas X-A Program Keahlian Akuntansi SMK Shalahuddin Malang, (Malang: Fakultas Teknik UM, 2007), hal. 20
ثانياً، إدراك معنى الوضع برمّته بواسطة الرموز

1). القدرة على تغيير رمز الكتابة إلى

$$
\text { الصوت }
$$

الأبجدية العربية لديها نظام مختلف عن

الأبجدية اللاتينية. الأبجدية العربية هي "sillabary" "alphabetic". . وهناك فرق آخر هو نظام الكتابة العربية الذي يبدأ من اليمين إلى اليسار، والأحرف الكبيرة غير المعروفة بنمط معين لبدء جملة جديدة، وكتابة الاسم الشخ ل أو المكان، والأشكال المختلفة للحروف العربية عند الوقوف وحدها، في البداية، وفي المنتصف وفي النهاية. Y). إدراك فهم معنى القراءة هذا الجانب هو في قلب القراءة. هناك ثلاثة عناصر يجب النظر فيها وتطويرها في دروس القراءة لهذا الفهم، أي عناصر Pengajaran......, hal. 127 
كل دورة من أربعة مراحل: (1) التخطيط،

(r) التنفيذ، (r) المراقبة، (ع) التفكير. بسبب تحديد عدد الدورات يجب أن يرى الباحث معدل نجاح الدورة التي تم تنفيذها. إذا وصلت العملية التعليمية في الدورة الأولى إلى الهدف المتوقع، استخدم الباحث دورة واحدة فقط. ولكن لو في الدورة الأولى أوجه قصور تحتاج إلى تحسين، فيحتاج الباحث إلى مواصلة الدورة التالية. ب ـ مجتمع الدراسة تم إجراء هذا البحث في الجامعة معارف الإسلامية هضة العلماء مترو لمبونج في الفصل الرابع. كانت موضوعات الدراسة طلبة الفصل الدراسي الرابع، البالغ عددهم |لاطالباً. نظرًا لأن هذا البحث عبارة عن بحث الإجرائي، فإن الموضوعات التى تتم تناوها في فصل واحد فقط. r. (- مأدوات الدراسة تجمع نوع البيانات في هذا البحث إلى قسمين، هما: البيانات الرئيسية التي تغطي
تنظيم شروط ممارساقم التعليمية، والتعلم من تجاربهم الخاصة. تتم المحاولة بحث الإجرائي كفكرة للتحسين في ممارسة التعلم ويرى التأثير الحقيقي للجهد. بحث الإجرائي هو دراسة تعكس من قبل الجهات الفاعلة التي التخذت لتحسين القدرة العقلانية للإجراءات التي تقوم بها، وتحسين الظروف التي تجري في ظلها محارسات التعلم، لذا فإن الغرض من هذا بحث الإجرائي هو تحسين تنفيذ الممارسة في عملية التعلم من خلال تحسين وسائل الإعلام، والطريقة، واستراتيجيات التعلم حيث من المتوقع أن يؤدي التحسن إلى تحسين نتائج تعلم $\wedge$

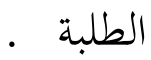
تنفيذ البحث الإجرائي في الفصول الدراسية من خلال عدة دورات حيث تتكون Tindakan Kelas, (Malang:Um Press, 2008), hal. 15 
المراقبة، (د) مرحلة التفكير. ب. ب الدورة الثانية بعد التفكير في الدورة الأولى، يمكن الاستمرار في الدورة الثانية إذا لم يتم تحقيق إتقان التعلم سواء بشكل فردي أو كاسيكي. في الدورة الثانية، تكمل أساسا ما هو مفقود وتحتاج إلى تحسين في الدورة الأولى لإنتاج النتيجة النهائية أو النتيجة البحث. تنفيذ الدورة الثانية مثل الدورة الأولى ويتم إجراؤها

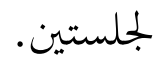

\section{نتائج البحث}

ا.تطبيق تعليم القراءة النصوص العربية بالإستراتيجية المعرفية مع تقنية أخذ الملاحظات لدي الطلبة الفصل الرابع بجامعة معارف الإسلامية هضة العلماء

$$
\text { مترو لمبونج }
$$

تم إجراء هذا البحث بدورتين، كل حرير
يتم القيام به من خلال أربعة مراحل: (1)
إجراء التخطيط، (r) تنفيذ العمل، (r) (ب) في الدورة الأولى أربعة مراحل: (أ) مرحلة التخطيط، (ب) مرحلة التنفيذ، (ج) مرحلة

قيمة الاختبارات السابقة (pretes) ونتائج الاختبار لكل دورة (postes) والبيانات الداعمة، بما في ذلك استجابة الطلبة بعد الاستراتيجية الإدراكية المطبقة مع تقنية أخذ القيمة النسبة نقاط الربح

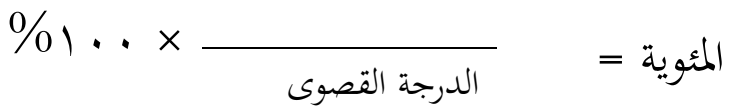
الملاحظات في شكل أوصاف وصفية مستمدة من المقابلة. الملاحظات الميدانية، وصحة المحاضر في تطبيق خطط الدروس، وأنشطة الطلبة أثناء أنشطة التعلم مع تقنية أخذ الملاحظات. تشمل مصادر البيانات في هذا البحث لطلبة تعليم اللغة العربية في الفصل الرابع الذي بلغ الطالباً في الجامعة معارف الإسلامية فضة العلماء مترو لمبونج. ع. أسلوب تحليل البيانات 
الأقصى للنتيجة هو 10 استناداً إلى بيانات

الملاحظة، و (ع ) التفكير. المراقبة، النسبة المئوية لمتوسط الدرجات هو أ. نتائج تنفيذ الدورة الأولى - م 1 ) التيجة الملاحظة نشاط المحاضر أثناء عملية مع تضمين القدرة على الإتقان في الفئة"

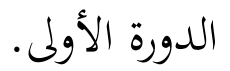
تحليل البيانات الملاحظة باستخدام تحليل ب ". على أساس وصف الجدول، في تطبيق النسبة المئوية، مع الدرجة المؤهل جيد جدا هذا التعليم، يكون الباحث أكثر هيمنة ع، الدرجة الجيدة ب، البجرد التسجيل ب، باستخدام خطوة تعلم المحاضرة، مما يؤدي إلى وأقل الدرجة ا ـ تتم جمع الدرجات التي عدم التفاعل بين الطلبة. r. نتيجة المالاحظة للنشاط الطلبة أثناء عملية التعلم مع تقنية أخذ الملاحظات في

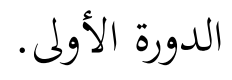

بالإضافة إلى ملاحظات أنشطة المحاضر، من ملاحظة المراقبة أن المحاضر هو نشاط يستخدم تحليل البيانات لنتائج الرصد الخاصة المحاضر الذي هو الباحث في الإستراتيجية بأنشطة الطلبة أيضًا تحليل النسبة المئوية، مع المعرفية مع تقنية أخذ الملاحظات في عمل الحصول على الدرجة المؤهل جيد جدا ع، الدورة الأولى، فإن مجموع الدرجات التي تم الدرجة الجيدة ب، المجرد التسجيل ب، وأقل الحصول عليها هو 0ـ في حين أن الحد الدرجة ا. يتم جمع النتائج التي تظهر لكل 
واصف وتسمى النتيجة بجموع النقاط. ثم استنادًا إلى واصفات الطاولات، يكون الطلبة تحسب متوسط القيمة باستخدام الصيغة كما من المعرفة المبكرة الأقل تطوراً التي تم الحصول عليها مسبقًا وكذلك أقل اهتمام بشرح المحاضر. بالإضافة إلى ذلك أن بعض الطلبة الذين لا يريدون تسجيل المفردات الصعبة التي طلبوها. ولكن في الأساس الطلبة جيدون بما فيه الكفاية في متابعة أنشطة التعلم مع خطوات التعلم المستخدمة بالمحاضر. سيتم تحسين بعض هذه الأشياء بواسطة الباحث في

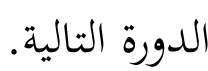

r.مرحلة التحليل والتفكير في العمل دورة

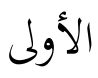

واستنادا إلى ملاحظات المحاضر من الشركاء، في الدورة الأولى من هذا النشاط كان المحاضر جيد وظهرت وجود ممارسات التعلم التي تتم وفقا للتخطيط المخطط للتعلم من ملاحظة المراقبة أن المحاضر الشريك

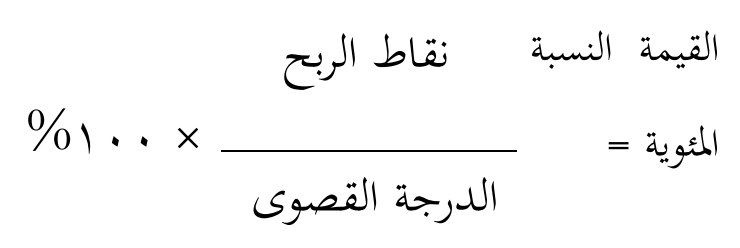

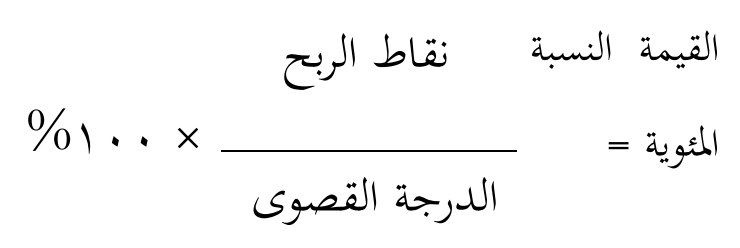
يلي: الحد الأقصى لدرجة الطلبة: 00ه لنشاط الطلبة في إجراء الدورة الأولى، فإن عدد الدرجات التي تم الحصول عليها هو · ع في حين أن الحد الأقصى للنتيجة هو استناداً إلى بيانات المراقبة، فإن النسبة المئوية لمتوسط النقاط هي الذي تم إعداده. ومع ذلك، هناك بعض السجلات التي تحتاج إلى تحسين. جلسة قراءة ونتيجة لذلك تم تضمين المستوى الجهرية تستغرق وقتًا طويلاً نظرًا لأن الطلبة التفاعلي للطلبة في عملية التعلم في فئة جيد. 
يُطلبمنهم تكرار قراءة غير الجهرية. هذا يجعل الطلبة الذين ما زالوا أقل حماسًا في اتباع الطلبة تبدو مشبعة ومتعبة. ومن المتوقع في التقنية التعليمية التي أجراها المعلم. قد يرجع الدورة القادمة أن يتم توقع ذلك من خلال ذلك إلى أن الطلبة ليسوا على دراية بعد التأكيد للطلبة على الاستعداد بشكل أفضل بتغيرات التعلم التي لم يسبق تطبيقها في التعليم في القراءة الجهرية. وبالمثل، فإن التغير في اللغة العربية. ب. نتائج تنفيذ الدورة الثانية التعلم وتخصي $\square$ الوقت المستخدم لكل مرحلة. يتم ذلك في محاولة لخلق جو تعلم متع ا.نتيجة الملاحظة نشاط المحاضر أثناء عملية دائمًا. التعلم مع تقنية أخذ الملاحظات.

تحليل البيانات الملاحظة باستخدام تحليل النسبة المئوية، مع الدرجة المؤهل جيد جدا

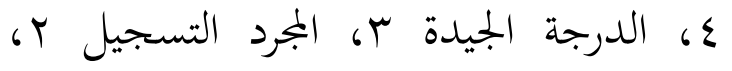
وأقل الدرجة ا. تتم جمع الدرجات التي تظهر لكل و واصفات و والنتيجة تسمى النتيجة الإجمالية. ثم تحسب متوسط القيمة باستخدام الصيغة كما يلي: وبناءً على الملاحظة، يمكن ملاحظة أن متوسط درجات الاختبار التي تشمل التمكن من فهم النصوص العربية قبل تطبيق الإستراتيجية المعرفية مع تقنية أخذ الملاحظات

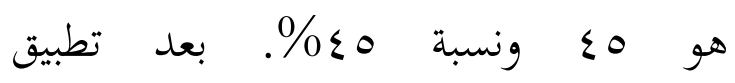
الإستراتيجية المعرفية معتقنية أخذ الملاحظات، كانت النتيجة المتوسطة لدرجة الاختبار

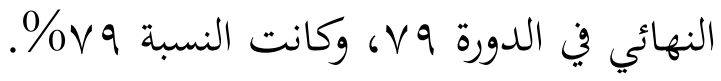
أقصى المحاضر الدرجة: وخل [0 الباحث في العمل اوباو بوفي نقاط الربح القيمة النسبة المئوية هذه الدورة الأولى إلى أن عملية التكيفية مع الطلبة جيدة وإن كان لا يزال هناك بعض 
Y. نتيجة الملاحظة للنشاط الطلبة أثناء عملية

التعلم مع تقنية أخذ الملاحظات في

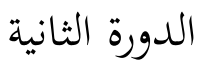

بالإضافة إلى ملاحظات أنشطة المحاضر،

يستخدم تحليل البيانات لنتائج الرصد الخاصة

بأنشطة الطلبة أيضًا تحليل النسبة المئوية، مع

الحصول على الدرجة المؤهل جيد جدا ع،

الدرجة الجيدة r، المجرد التسجيل ب، وأقل

الدرجة ا ـ تتم جمع النتائج التي تظهر لكل

واصف وتسمى النتيجة بجموع النقاط. ثم

تحسب متوسط القيمة باستخدام الصيغة كما

يلي:

الحد الأقصى لدرجة الطلبة: به

القيمة النسبة نقاط الربح

$\% 1 \cdots \times$

$$
\text { المئوية = }
$$

من نتيجة الملاحظة المحاضر الشريك

كمراقب لنشاط الطلبة في الإجراء الدورة
من ملاحظة المراقبة أن المحاضر هو نشاط

المحاضر الذي هو الباحث في الإستراتيجية

المعرفية مع تقنية أخذ الملاحظات في إجراء

الدورة الثانية، تكون الدرجة الكلية هـ بينما

تكون الدرجة القصوى ه V بناءً على بيانات

الملاحظة، النسبة المئوية لمتوسط الدرجات هي

«o

$$
\% \wedge \cdot=\% 1 \cdots \times \frac{}{70}
$$

مع تضمين القدرة على الإتقان في الفئة "أ". واستناداً إلى واصف الجدول، كان الباحث في تطبيق التعلم جيد جدًا في تقديم الموضوع أيضًا في مادة القراءة مقلدة بشكل متكرر مع الصوت الجهرية. كما قام المحاضر (الباحث) أيضا بتحسين خطوة التعلم من خلال عدم وجود محاضرة رتيبة ولكن مع الأسئلة المتداولة، مما يجعل نمط التفاعل التعلم بشكل أفضل. 
الثانية، فإن عدد النقاط التي تم الحصول عليها جيداً وقد أظهر وجود مارسات التعليمية هو 0ـ بينما أن الحد الأقصى للنتيجة هو التتوافق مع التخطيط المخطط للتعلم الذي تم هـ استناداً إلى بيانات الملاحظة، فإن النسبة إعداده. كما يقوم الباحث كمحاضر بإجراء تحسين من نتيجة التفكير في الدورة الأولى، المئوية لمتوسط القيمة هي وذلك عن طريق التكييف الطلبة خلال العملية التعلم، وخاصة في جلسة القراءة الجهرية. يبدو الطلبة أكثر استعدادًا وليس

$$
\% \wedge r=\% 1 \ldots \times
$$
هذا استنادًا إلى الملاحظة المحاضر مشبعًا بسهولة/ متعب عند قراءة النشاط الشريك في العملية التعلم، يتم تضمين بصوت الجهرية. وبناءً على الملاحظات، يمكن الملاحظة المستوى التفاعلي للطلبة في العملية التعلم في أن المتوسط الدرجات الاختبار التي تشمل قدرة فهم النصوص العربية بعد تطبيق

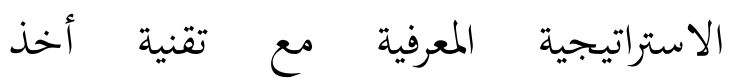
الملاحظات، فإن المتوسط النتيجة اختبار النتيجة النهائية في الدورة الثانية هي؟م،، والنسبة المئوية هي بحم\%. فئة جيد جلًا. يعتبر الطلبة جيد جلدًا في الالتفات إلى كل تفسير يقدمه المحاضر والطلبة أيضًا في تطوير المعرفة الأولية التي تم الحصول عليها مسبقًا. لذا فإن الطلبة تكونوا جيدا للغاية في المتابعة الأنشطة التعلم من خلال خطوات التعلم المستخدمة بالمحاضر. تظهر النتيجة الإجمالية للملاحظة في r. تحليل المرحلة والتفكير في الدورة الثانية واستناداً إلى ملاحظات المحاضر من الدورة الثانية أن الطلبة أصبحوا أكثر نشاطًا الشركاء، في الدورة الثانية، كان نشاط المحاضر وحريصًا على متابعة الدرس العربية. وفقا 
للمقابلات مع المحاضر الشريك وبعض معدل النجاح في الفئة "أ" وأيضاً على أساس الطلبة، في عملية العمل وبعد العمل، من التحليل البيانات المراقبة عن نشاط الطلبة المعروف أن أنشطة العملية التعليم والتعلم أظهر مستوى نجاح الجودة جيد جدا. في في سن تتزايد/ كلاهما من الدورة الأولى. ويتضح ذلك الدورة الثانية، يكون للباحث أقصى درجات

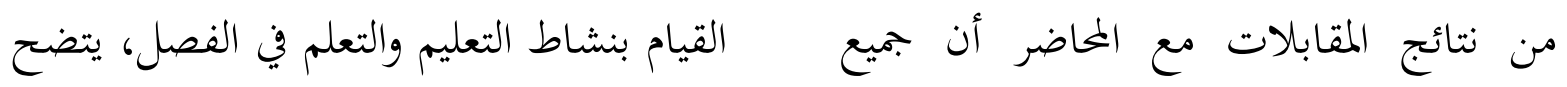
الطلبة متحمسون وأكثر تركيزًا في متابعة ذلك ملك من خلال جو الفصل الدراسي الهادئ

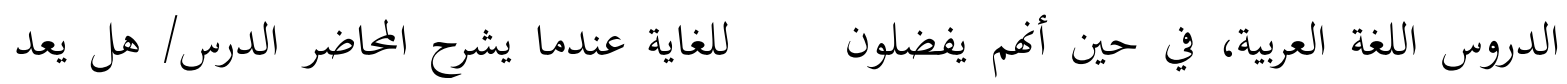
عدم الالتفات إلى المحاضر ولا يككنهم الإجابة الاختبار والجو أكثر حيوية عند إجراء الأنشطة الأسئلة والأجوبة. عن أسئلة المحاضر.

في تنفيذ الإستراتيجية التعلم المعرفية مع تقنية أخذ الملاحظات في هذه الدورة الثانية، نستنتج أن التمكن من فهم النصوص العربية للطلبة قد ازدادا. ويمكن الملاحظة من خلال التنييل المتوسط درجات النقاط للدورة الثانية

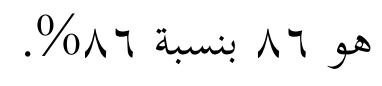

مع تزايد القدرة على فهم النصوص العربية لطلبة تعليم اللغة العربية في الفصل

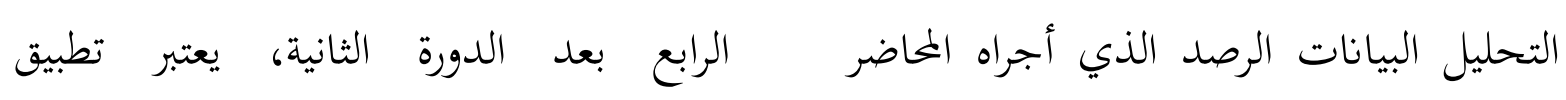

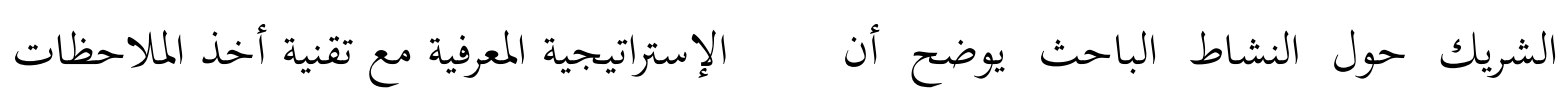

حين إجابات الطلبة حول انطباعهم عن عملية التعليم والتعلم التي تم تنفيذها، فإن معظمهم يشعرون أن العملية التدريس والتعلم أكثر متعة وأسهل في فهم المادة بعد تنفيذ

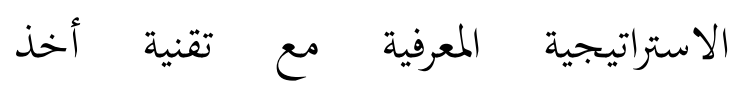

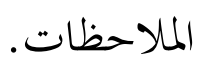
تم عمل التفكير لتحديد الإجراء الخاص بالدورة الثانية قد نجحت أو لمُ يعتمد على 
النشرات الكاملة في الملحق. قدم لفترة وجيزة

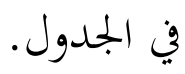

الجدول. النسبة المئوية للملصقات الطلبة

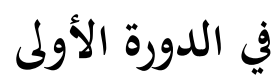

\begin{tabular}{|c|c|c|c|}
\hline النسبة المئوية & النسبة & نتيجة المتوسط & عدد \\
\hline لإتقان & المئوية & الدرجة & الطلبة \\
\hline$\% \wedge \vee$ & $\% \vee$ & V & 11 \\
\hline
\end{tabular}

وبناءً على الجدول أعلاه، الملاحظة في

المرحلة الأولى من طلبة الفصل الرابع الذي بلغ

$$
111
$$

99. ومع ذلك، لا يزال هناك طالبين لم يكملين الدراسة، بحيث تكون النتائج التي

تمت الحصول عليها نسبة إتقاها كNV.

Vي يكن تفسير متوسط النتائج الصف إلى

أن طلبة الفصل الدراسي الرابع يعتبرون تعلماً

$$
\text { كاملاً في تعليم اللغة العربية. }
$$

r ب الدورة الثانية
على مادة "الطعام والشرب" لترقية قدرة لطلبة في فهم النصوص العربية يعتبر كافيًا وتوقف حتى هذه الدورة الثانية. تستند دورة الإقلاع على نتيجة تقييم المنشورات وورقة الملاحظة التي يملؤها المحاضر الشريك. وقد لوحظت الزيادة في هذه الدورة الثانية، حيث زاد المتوسط القيمة قدرة القراءة العربية \مـ.

ج.نتيجة لترقية قدرة لطلبة في فهم النصوص العربية من خلال استخدام الاستراتيجية المعرفية مع تقنية أخذ الملاحظات استنادًا إلى نتائج التحليل الاختبار والمقابلات التي تمت إجراؤها في هاية كل الدورة، حصلت البيانات على ما يلي:

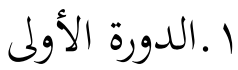
تم الحصول على بيانات النتائج لقدرة فهم النصوص العربية بعد تطبيق الإستراتيجية المعرفية مع تقنية أخذ الملاحظات من نتائج النشرات التي تمت في فاية دورة الأولى والتي يتبعها الطالبًا. يتم عرض البيانات نتائج 
تم الحصول على بيانات نتائج القدرة في بلغ به عامًا أن طلبة الفصل الدراسي الرابع فهم النصوص العربية بعد تطبيق الإستراتيجية يُعتبرون تعلماً كاملاً في تعليم اللغة العربية مع المعرفية مع تقنية أخذ الملاحظات من نتائج الحد الأدى من معايير الاكتمال التي تم النشرات التي تمت إجراؤها في فاية الدورة ت تحديدها في

استنتاج البيانات من المقابلات مع الطلبة الثانية والتي أعقبها 1 ا طالبًا. يتم عرض أنه من خلال الإستراتيجية المعرفية مع تقنية بيانات نتائج النشرات الكاملة في الملحق. أخذ الملاحظات، فهم قادرون على فهم قدم لفترة والجيزة في الجدول. النصوص العربية بسهولة أكبر. مع مساعدة الجدول. نسبة الملصقات للطلبة في الدورة من وسائل الإعلام بطاقة وأخذ الملاحظة الثانية المستخدمة في الدروس فإنه يساعدهم على فهم الخطاب/ النصوص العربية دون الحاجة إلى ترجمة التقنيات إلى الإندونيسية. مستوى فهمهم للنصوص العربية هو أعلى المقارنة مع تقنية أخذ الملاحظات.

r.مقارنة بين الدورة الأولى والدورة الثانية عدد نتيجة متوسط النسبة النسبة المئوية الطلبة الدرجة المئوية لإتقان $\% 90 \quad \% \wedge 7 \quad \wedge 7 \quad 11$ بناءً على الجدول أعلاه، الملاحظة في الدورة الثانية من الفصل الرابع، نجحت الطلبة استنادًا إلى نتائج المقارنة نتائج القيم في الدورتين الأولى و الثانية، فقد وجد أن نتائج فهم النصوص العربية قد ازدادت. وترد في الجدول المقارنة بين نتائج الطلبة لدورة الأولى الذين بلغوا 11 الدرجات بـ ـ. وقد تمت دراسة جميع الطلبة بدقة، مما أدى إلى نسبة اكتمال بنسبة ه\%\%. تفسير المتوسط النتائج الفصل الذي 


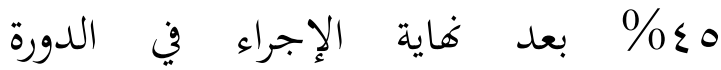

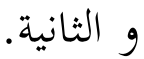

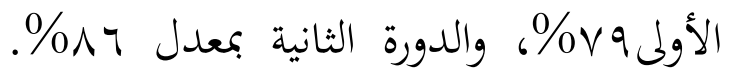
الزيادة هي حقيقة أن الاستراتيجية المعرفية مع تقنية أخذ الملاحظات أن ترقية قدرة لطلبة في فهم النصوص العربية.

\section{خلاصة}

\section{الجدول. متوسط مقارنة ملصقات الطلبة} في الدورتين الأولى و الثانية

\begin{tabular}{ccc}
\hline ترقيات & HP2 & HP1 \\
\hline$\% \vee$ & $\wedge 7$ & $\vee 9$
\end{tabular}

بعد تطبيق الإستراتيجية المعرفية مع

تقنبة أخذ الملاحظات ظهرت النتائج: (1)

زيادة قدرة لطلبة في فهم النصوص العربية مع تقنية أخذ الملاحظات. في الإجتماع الأولى من الدورة الأولى التي عرفتها في جلسات القراءة الجهرية، كان معظم الطلبة أقل مجموعًا في التصويت، ولكن في الاجتماع الثاني زاد. (Y) يشعر الطلبة أيضًا بالمساعدة في فهم محتويات النصوص العربية مع تقنية أخذ الملاحظات لأن هذه التقنية بسيطة جدًا وسهلة التطبيق.(r) من البيانات التي تم الحصول عليها، من المعروف أن قدرة لطلبة فن فهم النصوص العربية قد ازدادت، أي قبل إجراء نتائج تعلم الطلبة في المتوسط فقط

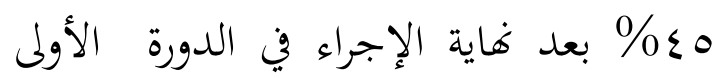

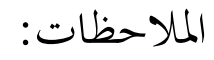

HP1

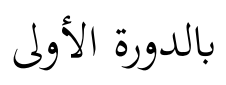

HP2

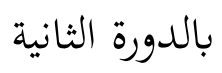

استناداً إلى الجدول أعلاه، الملاحظة أن متوسط النسبة المئوية للملصقات النابتحة لطلبة فن فهم النصوص العربية قد زادت من الدورة الأولى إلى الدورة الثانية وهي تساوي V\%\%. شهلت كل الدورة الزيادة كبيزة أي قبل إجراء نتائج تعلم الطلبة في المتوسط فقط 
Should Know. USA: Newbury House Publishers.

Ristianah, Nina. (2007). Penerapan Metode Pembelajaran Model Teams Games Tournament (TGT) Untuk Meningkatkan Hasil Belajar Akuntansi Siswa Kelas X-A Program Keahlian Akuntansi SMK Shalahuddin Malang. Malang: Fakultas Teknik UM.

Wahidmurni, \& Nur Ali. (2008). Penelitian Tindakan Kelas. Malang: UM Press.

Yamin, Martinis. (2008). Paradigma Pendidikan Konstruktivistik. Jakarta: Gaung Persada Press.

Fakhruddin, Agus Abid. (2009). Peningkatan Kemampuan Membaca Bahasa Arab Siswa Kelas VIII dengan Permainan Kartu "Rangkai" di SMP Plus Hidayatullah Mubtadiin Singosari Malang. Skripsi: Tidak di Terbitkan. Malang: FS UM.

Nugraha, E. S. (2008). Cara Membaca Yang Menyenangkan (online). (www.sekolahindonesia.com). diakses pada hari senin, tanggal 15 Januari, pukul 09.30

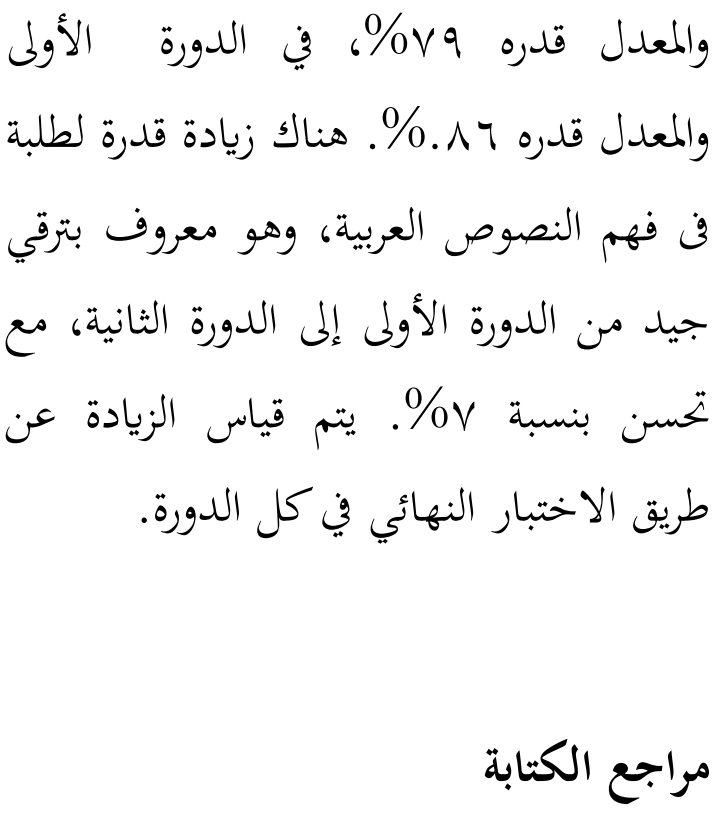

Ainin, M. \& Tohir, M. \& Asrori, Imam. (2006). Evaluasi dalam Pembelajaran Bahasa Arab. Malang: IKIP Malang.

Ainin, Moch. (2007). Metodologi Penelitian Bahasa Arab. Malang: Hilal Pustaka.

Al Abdullah, A. F. (2007). Asasu Ta'lim Al Qira'ah Al Naqidah $\mathrm{Li}$ Al Thalabah Al Mutafawwiqu:n Aqliyyan. Yordania: 'A:lim Al Kutub Al Hadits.

Al Khuli, M. A. (1986). Asa:li:b Tadri:s Al Lughah Al Arabiyah. Kairo: Jami:' Al Huquq.

Effendy, Fuad. (2009). Metodologi Pengajaran Bahasa Arab. Malang: Misykat.

Elliot, J. (1992). Action Research For Educational Change. Buckingham: Open University Press.

Nurhidayati, \& Anisah. (2005). Strategi Pembelajaran Bahasa Arab Untuk Anak. Malang: Fakultas Sastra UM.

Oxford, R. L. (1990). Language Learning Strategies. What Every Teacher 